\title{
Socioeconomic impact of traditional Korean medicine, Pyeongwee-San (KMP6) as an anti-allergic inflammatory drug
}

\author{
Young-Hoon Song ${ }^{1, \dagger}$, Sun-Young Nam ${ }^{1, \dagger}$, Youngjin Choi ${ }^{2}$, Jeong-Hwa Kim ${ }^{3}$, Young-Sick Kim ${ }^{1}$, Hyun-Ja Jeong ${ }^{2 *}$ \\ ${ }^{1}$ Department of Pharmacology, College of Oriental Medicine, Kyung Hee University, 1 Hoegi-dong, Dongdaemun-gu, Seoul, \\ 130-701, Republic of Korea; ${ }^{2}$ Biochip Research Center, Hoseo University, 165 Sechul-ri, Baebang-myun, Asan, Chungnam, 336-795, \\ Republic of Korea; ${ }^{3}$ Graduate School of Social Welfare, Ewha Womans University, 11-1 Daehyun-Dong, Seodaemun-Gu, Seoul, \\ 120-750, Republic of Korea
}

\begin{abstract}
The prevalence of allergic disease has been increasing over the past few decades in the majority of Western industrialized nations. There are some socioeconomic disparities regarding allergic disease status and management. Pyeongwee-San (KMP6) is Korean medicine for the treatment of gastrointestinal tract disease. It is known that KMP6 has an improving effect on the spleen and stomach functions in traditional Korean medical theory. Here, we hypothesized that KMP6 could be used to regulate the inflammatory reaction. We show the molecular mechanisms of Pyeongwee-San (KMP6) on inflammatory reactions. A molecular docking simulation showed that hesperidin, component of KMP6, regulate the enzymatic activity by interaction in the active site of caspase-1. KMP6 control the activity of caspase-1 in activated human mast cell line (HMC-1 cells). KMP6 reduced the expression of receptor interacting protein (RIP)-2 in HMC-1 cells. Thymic stromal lymphopoietin protein production and mRNA expression were inhibited by KMP6. In the activated HMC-1 cells, KMP6 suppressed the activation of mitogen-ativated protein kinase and nuclear factor-kappaB. In addition, KMP6 significantly inhibited the expression of inflammatory cytokines. Our findings indicate that KMP6 may attenuate allergic reactions via the regulation of caspase-1/RIP-2 signaling pathway. These studies will help advance the social welfare system.
\end{abstract}

Keywords caspase-1, hesperidin, molecular docking simulation, Pyeongwee-San (KMP6), thymic stromal lymphopoietin

\section{INTRODUCTION}

The prevalence of allergic disease has been increasing over the past few decades in several countries worldwide (Asher et al., 2006). 100 to 150 million people are suffering from asthma around the globe, including 6 million children in the United States alone (Camargo, 2009). Forty-eight percent of children have self-reported symptoms of allergic disease in Asia (Yao et al., 2011). Boys are more vulnerable to the allergic disorders such as asthma or rhinitis than girls (Yao et al., 2011). Low-income communities of African-American have higher rates of asthma than other communities (Ford et al., 1996). A recent study also demonstrates that children living in the inner city are more vulnerable to allergic disease due to high levels of indoor pollutants and allergens (Hansel et al., 2011).

Allergic diseases, defined as disorders of the immune system, affects approximately one third of the population in Western world and the costs associated with them, directly and indirectly, dominate public health budgets (Miyazaki et al., 2005). Allergic disease includes asthma, allergic rhinitis (AR), atopic dermatitis (AD), urticaria, conjunctivitis, food allergy, and severe anaphylactic responses. The central effector cells of

${ }^{*}$ Correspondence: Hyun-Ja Jeong

E-mail: hjjeong@ @oseo.edu

"The authors contributed equally to this work.

Received July 6, 2012; Accepted August 27, 2012; Published August 31,2012

doi: http://dx.doi.org/10.5667/tang.2012.0027

(C)2012 by Association of Humanitas Medicine

TANG / www.e-tang.org allergic disease are mast cells (Galli et al., 2008). Mast cells are involved in the progress of immediate type immune reaction as they release histamine, prostaglandins $\mathrm{D}_{2}$, leucotriene $\mathrm{C}_{4}$, chemokines, and cytokines such as interleukin (IL)-1 $1 \beta$, IL-6, or tumor necrosis factor (TNF)- $\alpha$ (Galli et al., 2008).

Thymic stromal lymphopoietin (TSLP) is associated with AR and AD (Bunyavanich et al., 2011; Ziegler et al., 2010). It might initiate Th2 polarization through an OX40-dependent mechanism that affects dendritic cells (DC) activity (Ziegler et al., 2010). TSLP also stimulates mast cells to produce interleukin (IL)-5, IL-6, IL-13, and granulocyte macrophage colony-stimulating factor (Allakhverdi et al., 2007). TSLP was expressed by tumor necrosis factor (TNF)- $\alpha$ and IL-1 $\beta$ in human airway smooth muscle cells via mitogen activated protein kinase (MAPK), p38, and extracellular signal-regulated kinase (ERK) signaling pathway (Zhang et al., 2007). Recently, we reported that TSLP was expressed and produced by caspase-1 and nuclear factor (NF)- $\mathrm{\kappa B}$ activation in mast cells (Moon et al., 2011).

Cystein-aspartic acid protease (caspase)-1 activation is mediated by cytosolic protein complexes termed inflammasomes, which function in various immune cells. Caspase- 1 is characterized by its ability to activate the inactive precursors of IL-1 $\beta$ and IL-18 (Lee et al., 2004). IL-1 $\beta$ is a proinflammatory cytokine which is proteolytically processed to its active form by caspase-1. The nuclear translocation of $N F-\kappa B$ is seen after the activation of mast cells by IL-1 $\beta$ (Lee et al., 2004). Therefore, caspase-1 could theoretically 
contribute to NF- $\kappa \mathrm{B}$ activation through the autocrine action of IL-1 $\beta$ on cell surface receptors (Wu et al., 2009)

Traditional Korean medicines were developed from simple drugs to combined drugs in order to cure diseases. The meanings the spleen and the stomach hold in Korean medicine are much more than that of the digestive organ system of modern medicine. Generally, the fact traditional drugs that strengthen the functions of the spleen and the stomach can be used to improve troubles that not only gastrointestinal problem, but also respiratory systemic mucosal inflammation such as rhinitis (Oh et al., 2012b). Based on it, old idea of Korean medicine that the spleen and stomach functions play an important role in the activities of the whole body. Recently, some studies reported that digestive tract disease is closely associated with allergic inflammatory diseases (Leung 2000). Therefore, in the current field of Korean medical clinics, many practitioners who treat allergic diseases apply treatments that improve the functions of the spleen and the stomach (Oh et al., 2011). Pyeongwee-San (KMP6) has frequently been used for the treatment of digestive tract disease as a traditional medicine in Korea. KMP6 has an improving effect on the spleen and stomach functions. Hesperidin, an active component of KMP6 potently regulates stem cell factor (SCF)-induced migration through the hindering of SCF and c-kit binding in mast cells (Jeong et al., 2011). Recently, we also reported that KMP6 has the regulatory effect in AR and AD animal model (Han et al., 2012; Oh et al., 2012a). However, it has not been sufficiently clarified how KMP6 and its active compound, hesperidin, modulate the responses of mast cells. Therefore, we examined whether KMP6 can regulate the TSLP production and caspase-1 activation in phorbol 12-myristate 13-acetate (PMA) and calcium ionophore A23187 (PMACI)-stimulated human mast cells (HMC)-1.

\section{MATERIALS AND METHODS}

\section{Materials}

PMA, A23187, dimethyl sulfoxide, 3-(4, 5-dimethylthiazol-2-yl)-2, 5-diphenyltetrazolium bromide, hesperidin, glycyrrhizin, dexamethasone, and other reagent were purchased from Sigma (St. Louis, MO, USA). Iacove's midified Dulbecco's medium (IMDM) and fetal bovine serum (FBS) were purchased from Gibco BRL (Grand Island, NY, USA). Anti-human TSLP, IL-1 $\beta$, IL- 6 , and TNF- $\alpha$ biotinylated anti-human TSLP, IL-1 $\beta$, IL-6, and TNF- $\alpha$, recombinant human (rh) TSLP, IL-1 $\beta$, IL-6, and TNF- $\alpha$ were purchased from Pharmingen (San Diego, CA, USA). Antibodies (Abs) for caspase-1, RIP-2, p38, pp38, JNK, pJNK, NF-кB, IкB $\alpha$, and b-actin were obtained from Santa Cruz Biotechnology (Santa Cruz, CA, USA). The caspase assay kit was supplied by R\&D Systems Inc. (Minneapolis, MN, USA).

\section{Cell culture}

HMC-1 cells have been established from the peripheral blood of a patient with mast cell leukemia (Butterfield et al., 1988). HMC-1 was grown in IMDM supplemented with $100 \mathrm{U} / \mathrm{ml}$ penicillin, $100 \mathrm{mg} / \mathrm{ml}$ streptomycin, and $10 \%$ heat inactivated FBS at $37^{\circ} \mathrm{C}, 5 \% \mathrm{CO} 2$ and $95 \%$ humidity.

\section{Preparation of KMP6}

KMP6 was provided by the Korea Medi Inc. (Seoul, Republic of Korea). KMP6 is composed of Atractylodes japonica Koidzumi (13.3 g), Magnolia officinale Rehder et Wils (10 g), Citrus sunki Hort. ex Tanaka (10 g), Zingiber officinale Roscoe (3.3 g), Glycyrrhiza uralensis Fisch (3.3 g), and Zizyphus jujuba var. inermis (Bunge) Rehder (6.7 g). The KMP6 was dissolved in distilled water (DW) and filtered with a $0.22 \mu \mathrm{m}$ syringe filter. We obtained the Pyeongwee-San, HS-PS (an over-the-counter drug for indigestion), from Han Kook Shin Yak pharmaceutical Co. (Nonsan, Republic of Korea) to compare with KMP6. HS-PS granules were prepared by dissolving in DW and being autoclaved for the sterilization and kept at $4^{\circ} \mathrm{C}$. HS-PS granules ( $\left.3.5 \mathrm{~g}\right)$ contained some excipients $(1.7 \mathrm{~g})$. We made the dose of HS-PS $(2 \mathrm{mg} / \mathrm{ml})$ two times stronger than KMP6 $(1 \mathrm{mg} / \mathrm{ml})$.

\section{ELISA}

Secreted TSLP, IL-1 $\beta$, IL-6, and TNF- $\alpha$ in culture supernatants were measured according to the manufacturer's specification (Pharmingen, San Diego, CA, USA)

\section{RNA isolation and RT-PCR}

Total RNA was isolated from HMC-1 according to the manufacturer's specifications using an easy-BLUE RNA extraction kit (iNtRON Biotech, Korea). Total RNA $(2.0 \mu \mathrm{g})$ was heated at $65^{\circ} \mathrm{C}$ for $10 \mathrm{~min}$ and then chilled on ice. Each sample was reverse-transcribed to cDNA for $90 \mathrm{~min}$ at $37^{\circ} \mathrm{C}$ using a cDNA synthesis kit. RT-PCR was carried out with $1 \mu \mathrm{l}$ of a cDNA mixture, in $20 \mu \mathrm{l}$ final volume with $2.5 \mathrm{mM} \mathrm{MgCl}_{2}$, $200 \mathrm{mM}$ dNTPs, $25 \mathrm{pM}$ cytokine primers, and $2.5 \mathrm{U}$ of TaqDNA polymerase in the reaction buffer $(50 \mathrm{mM} \mathrm{KCl}, 10$ $\mathrm{mM}$ Tris- $\mathrm{HCl}, \mathrm{pH} 9$, and $0.1 \%$ Triton X-100). PCR was performed with the following primers for human TSLP (5'-TAT GAG TGG GAC CAA AAG TAC CG-3' and 5'-GGG ATT GAA GGT TAG GCT CTG G -3'); IL-1 $\beta$ (5 CCG GAT CCA TGG CAC CTG TAC GAT CA 3; 5 GGG GTA CCT TAG GAA GAC ACA AAT TG 3); IL-6 (5 GAT GGATGC TTC CAATCT GGAT 3; 5 AGT TCT CCATAG AGA ACA ACA TA 3); TNF- $\alpha$ (5 CAC CAG CTG GTT ATC TCT CAG CTC 3; 5 CGG GAC GTG GAG CTG GCC GAG GAG 3); GAPDH (5'-CAA AAG GGT CAT CAT CTC TG-3' and 5'-CCT GCT TCA CCA CCT TCT TG-3'). Products were electrophoresed on a $1.5 \%$ agarose gel and visualized by staining with ethidium bromide.

\section{Molecular docking simulations}

Molecular docking simulation job was performed by Glide module in Maestro 9.2 software. All compounds were docked to caspase-1 in flexible mode. The three-dimensional coordinates for the caspase-1/z-VAD-FMK complex (PDB id 2HBQ) were downloaded from RCSB Protein Data Bank. To prepare the model system for caspase-1, z-VAD-FMK inhibitor structure was manually deleted. The protein was prepared for following grid generation using the Protein Preparation Wizard tool in the Maestro package. With this tool, all hydrogen atoms were added to caspase-1, protonation states were optimized, and structural waters near the heavy atoms were preserved. The modeled protein structure was further relaxed using energy-minimization method. Molecular grid was prepared for the caspase-1 using the Receptor Grid Generation tool in Glide with the $20 \AA$ of cubic box. To obtain accurate binding mode and affinity data, docking was conducted under $X P$ mode with Glide XP 5.0 scoring function. The highest-scoring docked pose for each ingredient with caspase-1 was ranked according to the Glide Score.

\section{Caspase-1 activity}

The enzymatic activity of caspase- 1 was assayed using a caspase colorimetric assay kit according to the manufacturer's protocol. The lysed cells were centrifuged at $15,000 \times \mathrm{g}$ for 5 min. The protein supernatant was incubated with $50 \mu$ reaction buffer and $5 \mu$ caspase substrate (WEHD-p-nitroaniline) at 
$37^{\circ} \mathrm{C}$ for $2 \mathrm{~h}$. The absorbance was measured was measured using a plate reader at a wavelength of $405 \mathrm{~nm}$. Equal amounts of the total protein from each lysate were quantified using a bicinchoninic acid protein quantification kit (Pierce, Rockford, IL, USA).

\section{Western blot analysis}

For analysis of protein level of indicated proteins in the text, stimulated cells were rinsed twice with ice-cold phosphate buffered saline (PBS) and then lysed in ice-cold lysis buffer (PBS containing $0.1 \%$ SDS, $1 \%$ triton and 1\% deoxycholate). Cell lysates were separated through electrophoresis, the protein was transferred to nylon membranes by electrophoretic transfer. The membranes were blocked in 5\% skim milk for $2 \mathrm{~h}$, rinsed and incubated overnight at $4{ }^{\circ} \mathrm{C}$ with primary Abs (Santa Cruz Biotechnology, Santa Cruz, CA). After three washes in PBS containing $0.05 \%$ Tween-20 (PBST), the membranes were incubated for $1 \mathrm{~h}$ with horse radish peroxidase-conjugated secondary Abs. After three washes in PBST, the protein bands were visualized by an enhanced chemiluminescence assay following the manufacturer's instructions.

\section{Transient transfection and luciferase assay}

The luciferase activity was measured according to the manufacturer's specification using Lipofectamine ${ }^{\mathrm{TM}} 2000$ (Invitrogen, Carlsbad, CA, USA). The relative luciferase activity was defined as the ratio of firefly luciferase activity to renilla luciferase activity.

\section{Statistical analysis}

Results were expressed as the mean \pm S.E.M. of independent experiments, and statistical analyses were performed by one-way analysis of variance with Tukey post hoc test to express the difference between groups. A value of $p<0.05$ was considered to indicate statistical significance.

\section{RESULTS}

Docking simulations of the components of KMP6 and caspase-1 interaction

Recently, we reported that caspase-1 plays an important role in allergic inflammatory reactions in mast cells (Moon et al.,
2011). To predict the caspase- 1 potentially active component in the KMP6 for caspase-1, docking simulations were performed using molecular docking software. Table 1 summarizes the final docking scores of each component bound to the caspase-1 active site. Caspase-1 inhibitor (z-VAD-FMK) was used as a positive control. The data for docking scores indicated that glycoside compounds including licuraside, hesperidin, glycyrrhizin, and poncirin were suitable binders for the caspase-1 structure because they ranked the highest docking score. KMP6 contains hesperidin about $5.26 \mathrm{mg} / \mathrm{g}$ and glycyrrhizin about $2.60 \mathrm{mg} / \mathrm{g}$ (data not shown). Hesperidin is a major component of KMP6. In this study, hesperidin was selected for a further evaluation after considering its applicability and attainability. We selected the concentrations of KMP6 $(0.01,0.1$, and $1 \mathrm{mg} / \mathrm{ml})$ and hesperidin $(10 \mu \mathrm{g} / \mathrm{ml})$ in accordance with our previous report (Jeong et al., 2011).

\section{Effect of KMP6 on caspase-1/RIP-2 expression in HMC-1 cells}

Binding mode of hesperidin-caspase complex was analyzed to understand the inhibitory mechanism of hesperidin at the atomic level. From the molecular docking simulations, hesperidin was complexed into the active site of caspase-1 like as z-VAD-FMK (Fig. 1A). Arrangement of the loop structure of caspase-1 surrounding hesperidin provided a stable docking site for this compound. In particular, caspase residue showed special inter-atomic contacts such as hydrophobic interaction or hydrogen bonding with the hesperidin (data not shown). Asp381, Arg383, and Trp340 of caspase-1 showed close atomic contacts with the flavonoid moiety of hesperidin. Hydrogen bonding was also found in Ser339, Arg341, and His 237 of caspase-1 with the sugar ring of the hesperidin (Fig. 1B). The docking study suggests that the planar structure of the flavonoid moiety of hesperidin is decisive factor to fix into crevasse form around the active site of caspase- 1 supported by hydrophilic contacts made by additional sugar residues in the hesperidin. To confirm the effect of hesperidin in vitro, we investigate the effect of KMP6 and hesperidin on caspase-1 activation induced by PMACI. Western blot analysis for caspase-1 was performed. Caspase-1 activation was significantly decreased with KMP6 and hesperidin treatment (Fig. 1C and D, $p<0.05$ ). We also measured the effect of KMP6 and hesperidin on caspase-1 activation using a

Table 1. Docking scores of the ranked poses for complexes between different components and the caspase-1 protein

\begin{tabular}{lcl}
\hline Component & Docking Score & Origin \\
\hline z-VAD-FMK & -14.222 & Caspase-1 inhibitor \\
Ac-YVAD-CMK & -8.539 & Caspase-1 inhibitor \\
Licuraside & -10.918 & Glycyrrhiza uralensis Fisch \\
Glycyrrhizin & -10.546 & Glycyrrhiza uralensis Fisch \\
Poncirin & -10.460 & Citrus sunki Hort. ex Tanaka \\
Hesperidin & -10.161 & Citrus sunki Hort. ex Tanaka \\
Liquiritin & -8.874 & Glycyrrhiza uralensis Fisch \\
Neoisoliquiritin & -6.884 & Glycyrrhiza uralensis Fisch \\
Isoliquiritigenin & -6.634 & Glycyrrhiza uralensis Fisch \\
myo-Inositol & -6.124 & Citrus sunki Hort. ex Tanaka \\
Betulic acid & -5.698 & Glycyrrhiza uralensis Fisch \\
Acetyl-atractylodinol & -5.545 & Atractylodes japonica Koidzumi \\
Oleanolic acid & -5.165 & Glycyrrhiza uralensis Fisch \\
Atractylenolide III & -5.132 & Atractylodes japonica Koidzumi \\
Magnolol & -4.903 & Magnolia officinale Rehder et Wils \\
Atractylenolide II & -4.731 & Atractylodes japonica Koidzumi \\
Coumarin & -4.692 & Glycyrrhiza uralensis Fisch \\
Atractylodin & -4.215 & Atractylodes japonica Koidzumi \\
d-Limonene & -4.138 & Citrus sunki Hort. ex Tanaka \\
Atractylenolide I & -4.135 & Atractylodes japonica Koidzumi \\
Magnocurarine & -3.825 & Magnolia officinale Rehder et Wils \\
Eudesmol & -3.824 & Magnolia officinale Rehder et Wils \\
Hinesol & -3.728 & Atractylodes japonica Koidzumi \\
Honokiol & -3.338 & Magnolia officinale Rehder et Wils \\
\hline TANG/
\end{tabular}


A

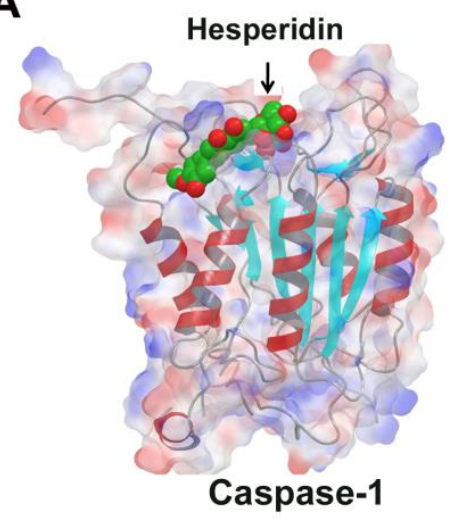

C

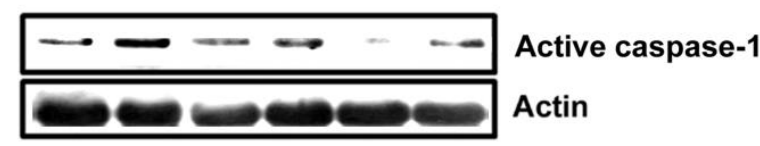

B

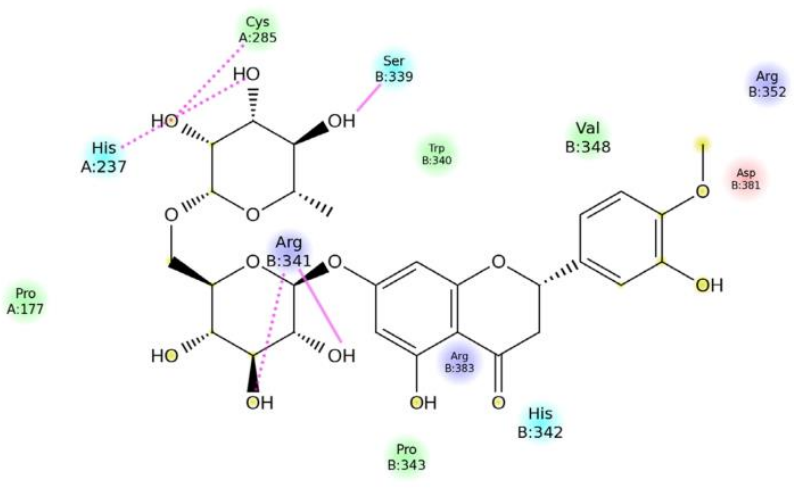

D

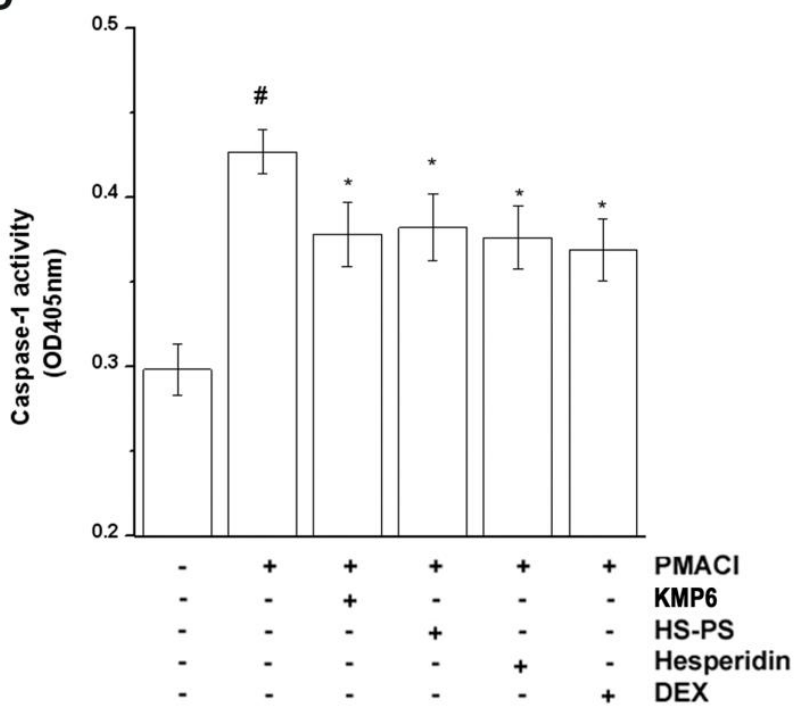

Fig. 1. Effect of KMP6 and hesperidin on RIP-2/caspase-1 activation in HMC-1. Molecular representations the docked pose of hesperidin complexed with caspase-1. (A) The hesperidin was bound to the active site of caspase-1. (B) Schematic interaction diagram within $3 \AA$ of a heavy atom of hesperidin showed characterized atomic the integrations between caspase-1 and hesperidin. (C) The cells were pretreated with of KMP6 (1 mg/ml), HS-PS $(2 \mathrm{mg} / \mathrm{ml})$, hesperidin $(10 \mathrm{mg} / \mathrm{ml})$, or dexamethasone $(10 \mathrm{nM})$ for $1 \mathrm{~h}$ prior to PMACI stimulation for 30 min. The levels of RIP-2 and caspase-1 were assayed by Western blot analysis. (D) The enzymatic activity of caspase-1 was tested by a caspase colorimetric assay. Results are representative of three independent experiments. ${ }^{*} p<0.05$ : significantly different from the PMACI-stimulated cells. \# $<0.05$ : significantly different from unstimulated cells. DEX, dexamethasone.

caspase-1 assay kit. Once again, this showed that caspase-1 activity was significantly decreased with KMP6 and hesperidin treatment (Fig. 1D, $p<0.05$ ). HS-PS and dexamethasone also significantly reduced the caspase- 1 activity $(p<0.05)$. RIP-2 expression induces caspase-1 oligomerization, and promotes its activation (Kimura et al., 2006). Then, caspase-1 induces pro-inflammatory cytokine maturation (Galli et al., 2008). We performed a Western blot analysis to determine the effect of KMP6 and hesperidin on RIP-2 expression induced by PMACI. The results indicated that KMP6, hesperidin, HS-PS, or dexamethasone suppressed RIP-2 expression induced by PMACI (Fig. 1C).

KMP6 inhibits TSLP protein production and mRNA expression

To assess the regulatory effects of KMP6 and hesperidin on TSLP secretion, cells were treated with KMP6 or hesperidin for $1 \mathrm{~h}$ prior to stimulation with PMACI for $8 \mathrm{~h}$. Culture supernatants were assayed for TSLP protein levels by the ELISA method. Dexamethasone $(10 \mathrm{nM})$ was used as a positive control. As shown in Fig.2A, KMP6, hesperidin, or HS-PS significantly inhibited the PMACI-induced TSLP secretion $(p<0.05)$. Cell cytotoxicity by KMP6, hesperidin, HS-PS, or dexamethasone was not observed (data not shown). We also performed a RT-PCR analysis for TSLP mRNA expression. The TSLP mRNA expression up-regulated by PMACI was decreased by the treatment with KMP6, hesperidin, HS-PS, or dexamethasone (Fig. 2B).

Effect of KMP6 on MAPK phosphorylation in HMC-1 cells The activation of MAPK induced TSLP expression (Choi et al., 2007). To determine the effect of KMP6 and hesperidin on MAPK activation induced by PMACI, Western blot analysis for phosphorylated $\mathrm{p} 38$ and JNK was performed. The cells were pretreated with KMP6, hesperidin, HS-PS, or dexamethasone for $1 \mathrm{~h}$ and then treated with PMACI for 30 min. The result showed that MAPK phosphorylation was increased by PMACI, but the treatment of KMP6, hesperidin, HS-PS, or dexamethasone suppressed the p38 and JNK phosphorylation in PMACI-stimulated cells (Fig. 3).

KMP6 inhibits NF-кB activation and IкB degradation

The expression of TSLP was regulated by the transcription factor, NF-kB (Faubel et al., 2007). KMP6 or hesperidin 


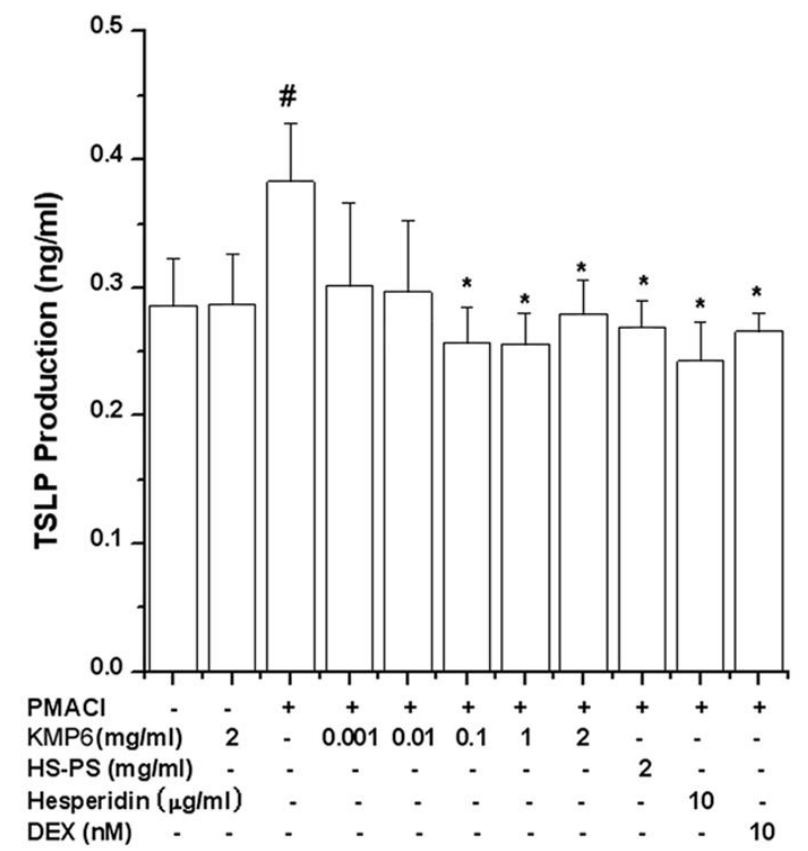

TSLP

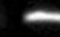

GAPDH

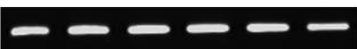

$\begin{array}{lllllll}\text { PMACI } & - & + & + & + & + & + \\ \text { KMP6 } & - & - & 1 & - & - & - \\ \text { HS-PS } & - & - & - & 2 & - & - \\ \text { Hesperidin } & - & - & - & - & 10 & - \\ \text { DEX } & - & - & - & - & - & 10\end{array}$

Fig. 2. Effect of KMP6 and hesperidin on TSLP secretion and mRNA expression in HMC-1. (A) The cells were pretreated with various concentrations of KMP6 $(0.001-2 \mathrm{mg} / \mathrm{ml})$, HS-PS $(2 \mathrm{mg} / \mathrm{ml})$, hesperidin $(10 \mathrm{mg} / \mathrm{ml})$, or dexamethasone $(10 \mathrm{nM})$ for $1 \mathrm{~h}$ prior to PMACI stimulation for $8 \mathrm{~h}$. Secreted TSLP levels were measured by ELISA method. (B) The cells were pretreated with KMP6 (1 mg/ml), HS-PS $(2 \mathrm{mg} / \mathrm{ml})$, hesperidin $(10 \mathrm{mg} / \mathrm{ml})$, or dexamethasone $(10 \mathrm{nM})$ for $1 \mathrm{~h}$ prior to PMACI stimulation for $5 \mathrm{~h}$. The total RNA was assayed by a RT-PCR analysis. Results are representative of three independent experiments. Data represent mean \pm S.E.M. of three independent experiments. $* p<0.05$ : significantly different from the PMACI-stimulated cells. ${ }^{\#} p<0.05$ : significantly different from unstimulated cells. DEX, dexamethasone.

decreased the expression level of NF- $\mathrm{kB}$ (p65) in the nucleus (Fig. 4A). To examine whether the inhibitory action of KMP6 or hesperidin was due to its effects on I $\mathrm{B}$ degradation, we investigated the cytoplasmic levels of IKB protein with a Western blot analysis. The expression level of I $\mathrm{KB}$ in the cytoplasm was increased by the treatment with KMP6 or hesperidin. Next, we examined whether KMP6 or hesperidin

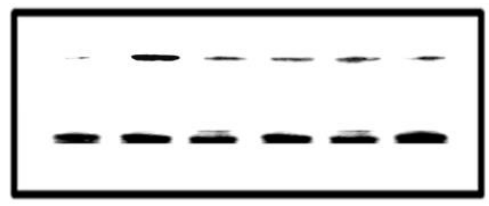

\section{phospho-p38 p38}

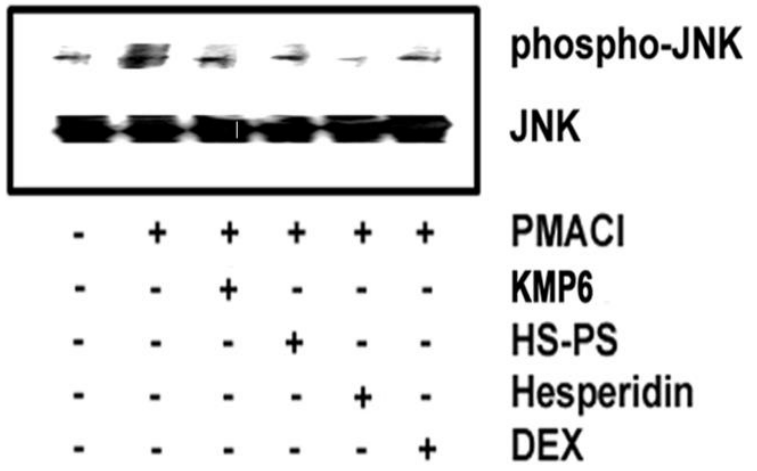

Fig. 3. Effect of KMP6 and hesperidin on MAPK phosphorylation in HMC-1. The cells were pretreated with of KMP6 $(1 \mathrm{mg} / \mathrm{ml})$, HS-PS (2 $\mathrm{mg} / \mathrm{ml})$, hesperidin $(10 \mathrm{mg} / \mathrm{ml})$, or dexamethasone $(10 \mathrm{nM})$ for $1 \mathrm{~h}$ prior to PMACI stimulation for $30 \mathrm{~min}$. The phosphorylated MAPK levels were assayed by Western blot analysis. Results are representative of three independent experiments. DEX, dexamethasone could modulate the luciferase expression specifically via $\mathrm{NF}-\kappa \mathrm{B}$ activation. As shown in Fig. 4B, the increased activity was significantly decreased by the treatment with KMP6 or hesperidin $(p<0.05)$.

Effect of KMP6 on inflammatory cytokines secretion and mRNA expression in HMC-1 cells

To assess the regulatory effect of KMP6 and hesperidin on IL-1 $\beta$, IL-6, and TNF- $\alpha$ secretion, we treated HMC-1 cells with PMACI for $8 \mathrm{~h}$ and we used the ELISA to analyze the supernatants for cytokines. The treatment with PMACI increased the IL-1 $\beta$, IL- 6 , and TNF- $\alpha$ secretions from HMC-1 cells. KMP6 and hesperidin significantly inhibited PMACI-induced IL-1 $\beta$, IL-6, and TNF- $\alpha$ secretions (Fig. 5A-C, $p<0.05)$. The maximal inhibition rate of IL-1 $\beta$, IL-6, and TNF- $\alpha$ secretions by KMP6 $(1 \mathrm{mg} / \mathrm{ml})$ were about $99.86 \%$, $35.05 \%$, and $75.90 \%$ respectively. The inhibitory effect of KMP6 $1 \mathrm{mg} / \mathrm{ml}$ is higher than that of KMP6 $2 \mathrm{mg} / \mathrm{ml}$ on cytokine secretion. HS-PS $(2 \mathrm{mg} / \mathrm{ml})$ significantly inhibited the IL-1 $\beta$, IL-6, and TNF- $\alpha$ secretions (about $100.10 \%$ for IL-1 $\beta$, $37.02 \%$ for IL- $6,35.10 \%$ for TNF- $\alpha, p<0.05$ ). The inhibitory effect of KMP6 $(1 \mathrm{mg} / \mathrm{ml})$ is similar to the effect of HS-PS on inflammatory cytokine secretion. Dexamethasone also significantly inhibited the inflammatory cytokine secretion $(p<$ $0.05)$. Using the pretreated HMC-1 cells described above, we also performed a RT-PCR analysis for IL-1 $\beta$, IL-6, and TNF- $\alpha$ to determine whether KMP6 modulated PMACI-induced cytokines expressions. IL-1 $\beta$, IL- 6 , and TNF- $\alpha$ mRNA expressions were up-regulated by PMACI stimulation but the up-regulated cytokine mRNA expressions were decreased with KMP6, hesperidin, HS-PS, or dexamethasone treatment (Fig. 5D).

2012 / Volume 2 / Issue 3 / e29 
A
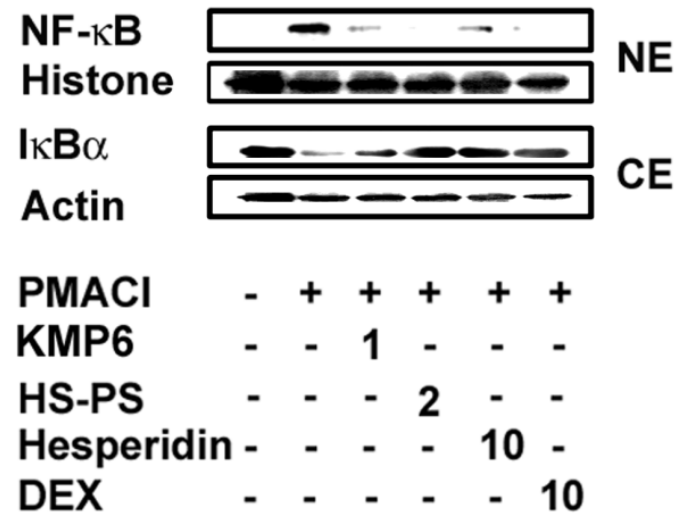

B

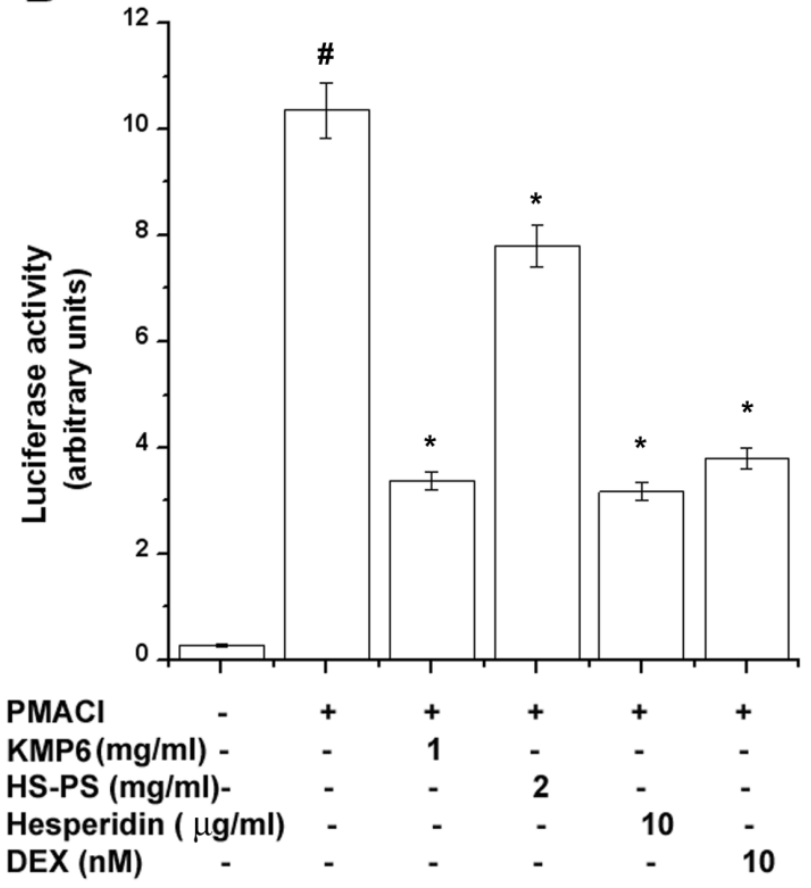

Fig. 4. Effect of KMP6 and hesperidin on NF- $\mathrm{BB}$ activation in HMC-1. The cells were pretreated with of KMP6 (1 mg/ml), HS-PS (2 mg/ml), hesperidin $(10 \mathrm{mg} / \mathrm{ml})$, or dexamethasone $(10 \mathrm{nM})$ for $1 \mathrm{~h}$ prior to PMACI stimulation for $2 \mathrm{~h}$. (A) Nuclear protein and cytoplasmic protein were prepared and analyzed for NF- $\kappa \mathrm{B}$ and I $\kappa \mathrm{B}$ by Western blotting as described in the experimental procedures. (B) The NF- $\kappa \mathrm{B}$ activity was measured by a luciferase assay. Results are representative of three independent experiments. ${ }^{*} p<0.05$ : significantly different from the PMACI-stimulated cells. ${ }^{\#} p$ $<0.01$ : significantly different from unstimulated cells. DEX, dexamethasone; NE, nuclear extract; CE, cytoplasmic extract.

\section{DISCUSSION}

The symptoms of allergic diseases affect individual's life in many ways, to name a few, activity restriction, sleep deprivation, reduced school attendance rate, poor work performance, feeling isolation and depression (Miller and Cohen, 2001). Asthma is the leading cause of activity limitation. People with asthma are reported to experience more than 100 days of restricted activity annually (Bailey et al., 1992). The condition of allergic diseases exerts a significant economic burden on society due to its impact on quality of life and the cost of treatment. The direct medical costs for allergic rhinitis (AR) in the US are estimated about \$US2-5 billion, and indirect costs estimates vary from \$US1.7 billion to \$US 6.3billion depending on assumptions for work productivity losses in 2003 values. (Reed et al, 2004).

Allergic diseases have developed from complex interactions between genes and environment. Allergic diseases, including a number of chronic diseases with no cure, currently affects almost one-fifth of the population of industrialized nations. Treatment can be challenging for physicians and patients alike. In previous studies, we systematically investigated the effects of various traditional medicines on allergic inflammatory reactions. Herbal remedies and botanicals are widely used by humans for both preventative and curative purposes. Traditional medicinal herbs have many benefits, few (if any) side effects, and display low cytotoxicity, and so the search for the use of natural products in traditional medicine is currently attracting intense interest. As part of our continuing search for clinically effective anti-allergic inflammatory agents from oriental medicines, we investigated KMP6 as a basic prescription of digestive disorders. Recently, we reported that digestive tract disease is closely associated with allergic disease (Han et al., 2011). KMP6 consists of 6 different herbs. Atractylodes rhizoma has been used for eliminating dampness, strengthening the spleen, expelling wind, and clearing away cold in traditional Korean medicine for centuries (Kimura et al., 2006). As for the chemical constituents of the Atractylodes rhizoma, many sesquiterpenoid glycosides and essential oils were reported (Kitajima et al., 2003). It contains sesquiterpenoids (1.5-3.0\% by weight), such as atractylenolide -I, -II, and -III, atractylon, and $3 \hat{a}$-acetoxyatractylon, as well as polyacetylene compounds, such as $(6 E, 12 E)$-tetradecadiene-8,-10-diyne-1,3-diol mono acetate, $\quad(6 E, 12 E)$-tetradecadiene-8,10-diyne-1,3-diol, and 6-methyl-2-geranyl-p-benzoquinone (Sakurai et al., 1993). Kang et al. (2011) found that atractylenolide-III had an anti-inflammatory effect. Citrus unshiu is minister medicinal of KMP6 as called 'Jinpi' in Korea. Citrus unshiu has many ingredients such as hesperidin, limonene, naringin, nobiletin, b-sitosterol, stigmasterol, Terpinen-4-ol, and vitamin C. Hesperidin (approximately 4.5 - 10\%), a main component of Citrus unshiu, has been reported to exhibit anti-inflammatory and antioxidant effects (Arafa et al., 2009; Choi et al., 2007). In addition, previous studies have revealed that citrus flavonoids such as naringin and nobiletin were found in anti-cancer, cardiovascular and anti-inflammatory activities (Benavente-García et al., 2008). Magnoliae cortex is the assistant medicinal of KMP6 and called 'Hubak' in Korea. Main compounds of Magnoliae cortex are known to magnolol, honokiol, and machiol. Magnolol is recently prime component for the efficacy in the inflammation via NF-kB activation (Tanaka et al., 2007). Moreover, magnolol also has the suppressive effect of various cancer cell proliferations as a chemoprevention therapeutic agent (Lee et al., 2009). Glycyrrhizae radix is a famous herbal in traditional Korean medicine because it contains the component of the prescription a lot, as one of the most active research. Glycyrrhizae radix includes glycyrrhzin, flavonoid, saponin, coumarin, essential oil, phytosterol, tannin, and other substances. Glycyrrhizin 
A

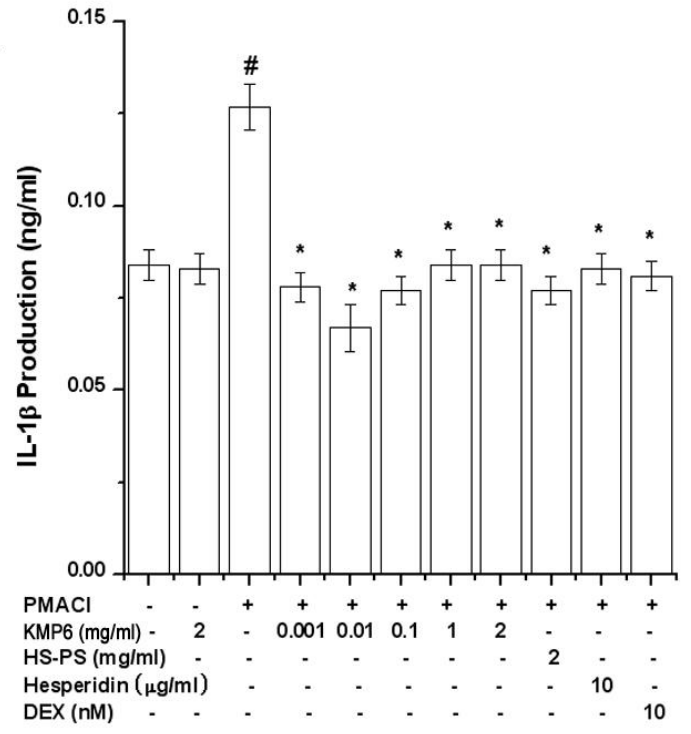

C

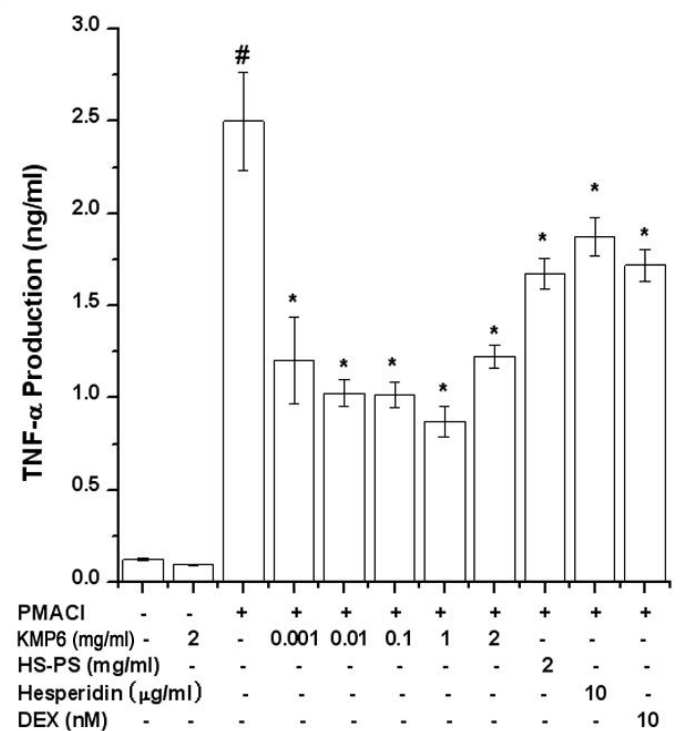

B

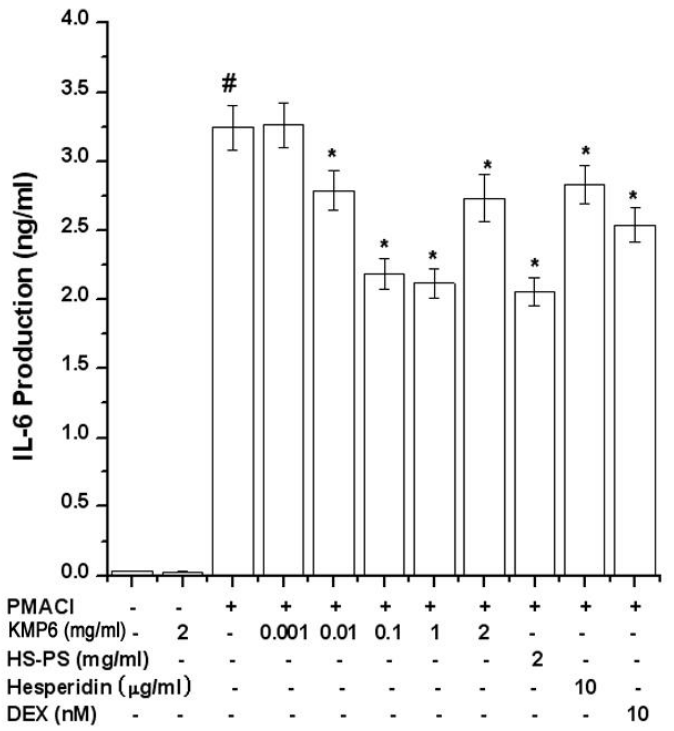

D

IL-1 $\beta$

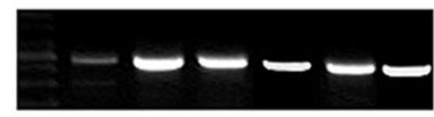

IL-6

TNF- $\alpha$

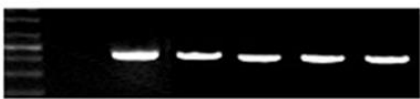

GAPDH

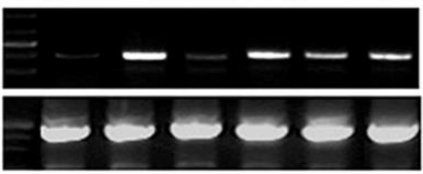

PMACI

KMP6

HS-PS

Hesperidin DEX

Fig. 5. Effect of KMP6 and hesperidin on inflammatory cytokine secretion and mRNA expression in HMC-1. (A-C) The cells were pretreated with various concentrations of KMP6 (0.001 - $2 \mathrm{mg} / \mathrm{ml})$, HS-PS $(2 \mathrm{mg} / \mathrm{ml})$, hesperidin $(10 \mathrm{mg} / \mathrm{ml})$, or dexamethasone (10 nM) for $1 \mathrm{~h}$ prior to PMACI stimulation for $8 \mathrm{~h}$. Secreted cytokine levels in culture supernatants of cells were measured by the ELISA method. (D) The cells were pretreated with KMP6 (1 mg/ml), HS-PS ( $2 \mathrm{mg} / \mathrm{ml})$, hesperidin $(10 \mathrm{mg} / \mathrm{ml})$, or dexamethasone $(10 \mathrm{nM})$ for $1 \mathrm{~h}$ prior to PMACI stimulation for $5 \mathrm{~h}$. The total RNA was assayed by an RT-PCR analysis. Results are representative of three independent experiments. Data represent mean \pm S.E.M. of three independent experiments. ${ }^{*} p<0.05$ : significantly different from the PMACI-stimulated cells. ${ }^{*} p<0.05$ : significantly different from unstimulated cells. DEX, dexamethasone.

(approximately $6-10 \%$ ), a major ingredient of Glycyrrhizae radix has chemopreventive activities on tumor promotion (Rahman et al., 2007) and anti-inflammatory effects (Takei et al., 2006). In this study, KMP6 and hesperidin inhibited PMACI-induced inflammatory cytokine expression on HMC-1 cells. KMP6 and hesperidin also inhibited MAPK activation. Therefore, we suggest that KMP6 has an anti-inflammatory effect by preventing the inflammatory reaction pathway.

Recently, Taylor et al. (2009) reported that TSLP regulates intestinal immunity and inflammatory reaction in helminth infection and colitis. Miyata et al. (2008) reported that mast cells play a critical role in TSLP expression in the nasal epithelium in allergic rhinitis and subsequent development of the disease. TSLP, derived from epithelial cells, has been shown to be capable of activating CD11 myeloid DC to up-regulate costimulatory molecules, leading to the differentiation of CD4+ T cells into Th2 cells. Therefore, it plays a key role in the development of allergic diseases such as asthma or AD (Ziegler et al., 2006). We reported for the first time that KMP6 and hesperidin reduced TSLP production and expression. Therefore, we found that hesperidin is an active compound of KMP6 on TSLP expression in mast cells.

Caspase- 1 is a member of caspases with large prodomains and its activation is involved in apoptosis and inflammation 
(Faubel et al., 2007).The activation of caspase-1 regulates the inflammation via secretion of pro-inflammatory cytokines and recruitment of neutrophil (Faubel et al., 2007). It was reported that caspase-1- $/-$ mice have decreased the secretion of IL-6 after stimulation with lipopolysaccharide (Kuida et al., 1995). RIP-2 is a specific adaptor molecule, and regulates the activation of caspase-1 (Lee et al., 2001). Other study showed that RIP-2 knockout reduced secretion of the pro-inflammatory cytokines such as TNF- $\alpha$ and IL-6 (Kobayashi et al., 2002). These studies suggested that the activation of RIP2/caspase- 1 is an attractive target for therapeutic of inflammatory diseases. Caspase 1 induced the TSLP expression via activating the NF-kB (Moon et al., 2011). Therefore, we postulated that KMP6 and hesperidin mediate its effects at least partly through the suppression of RIP2/caspase-1/NF- $\mathrm{KB}$ activation. In this study, we confirmed that KMP6 and hesperidin suppressed the RIP-2, caspase-1, and NF- $\mathrm{BB}$ activation induced by PMACI. This result suggested that the inhibitory effects of KMP6 and hesperidin on TSLP secretion might be derived through the regulation of RIP2/caspase-1/NF- $\mathrm{KB}$ signal pathways.

Allergies are associated with impairment of health-related quality of life (HR-QoL), including physical, social, emotional functioning and well-being of patients (Parker-Oliver, 2005). In this study, we identified the new effect of KMP6. Therefore, KMP6 may help improve the quality of life in allergy patients.

\section{ACKNOWLEDGEMENTS}

This research was supported by Basic Science Research Program through the National Research Foundation of Korea (NRF) funded by the Ministry of Education, Science and Technology (2011-0027280).

\section{CONFLICT OF INTEREST}

The authors declare that there are no conflicts of interest.

\section{REFERENCES}

Allakhverdi Z, Comeau MR, Jessup HK, Yoon BR, Brewer A, Chartier S, Paquette N, Ziegler SF, Sarfati M, Delespesse G. Thymic stromal lymphopoietin is released by human epithelial cells in response to microbes, trauma, or inflammation and potently activates mast cells. J Exp Med. 2007;204:253-258.

Arafa HM, Aly HA, Abd-Ellah MF, El-Refaey HM. Hesperidin attenuates benzo [alpha] pyrene-induced testicular toxicity in rats via regulation of oxidant/antioxidant balance. Toxicol Ind Health. 2009;25:417-427.

Asher MI, Montefort S, Björkstén B, Lai CK, Strachan DP, Weiland SK, Williams H, ISAAC Phase Three Study Group. Worldwide time trends in the prevalence of symptoms of asthma, allergic rhinoconjunctivitis, and eczema in childhood: ISAAC Phases One and Three repeat multicountry cross-sectional surveys. Lancet. 2006;368:733-743.

Bailey WC, Clark NM, Gotsch AR, Lemen RJ, O'Connor GT, Rosenstock IM. Asthma prevention. Task force on research and education for the prevention and control of respiratory diseases. Chest. 1992;102:216S-231S.

Benavente-García O, Castillo J. Update on uses and properties of citrus flavonoids: new findings in anticancer, cardiovascular, and anti-inflammatory activity. J Agric Food Chem. 2008;56:6185-6205.

Bunyavanich S, Melen E, Wilk JB, Granada M, Soto-Quiros ME, Avila L, Lasy-Su J, Hunninghake GM, Wickman M, Pershagen G, O’Connor GT, Weiss ST, Celedón JC. Thymic stromal lymphopoietin (TSLP) is associated with allergic rhinitis in children with asthma. Clin Mol Allergy. 2011;9:1.

Butterfield JH, Weiler D, Dewald G, Gleich G.J. Establishment of an immature mast cell line from a patient with mast cell leukemia. Leuk Res. 1988;12:345-355.

Camargo CA Jr, Rachelefsky G, Schatz M. Managing asthma exacerbations in the emergency department: summary of the National Asthma Education and Prevention Program Expert Panel Report 3 guidelines for the management of asthma exacerbations. J Emerg Med. 2009;37:S6-S17.

Choi IY, Kim SJ, Jeong HJ, Park SH, Song YS, Lee JH, Kang TH, Park JH, Hwang GS, Lee EJ, Hong SH, Kim HM, Um JY. Hesperidin inhibits expression of hypoxia inducible factor-1 alpha and inflammatory cytokine production from mast cells. Mol Cell Biochem. 2007;305:153-161.

Faubel S, Lewis EC, Reznikov L, Ljubanovic D, Hoke TS, Somerset H, Oh DJ, Lu L, Klein CL, Dinarello CA, Edelstein CL. Cisplatin-induced acute renal failure is associated with an increase in the cytokines interleukin (IL)-1beta, IL-18, IL-6, and neutrophil infiltration in the kidney. J Pharmacol Exp Ther. 2007;322:8-15.

Ford, ME, Edwards G, Rodriguez JL, Gibson RC. Tilley BC. An empowerment-centered, church-based asthma education program for African American adults. Health and Social Work. 1996;21:70-75.

Galli SJ, Grimbaldeston M, Tsai M. Immunomodulatory mast cells:negative, as well as positive, regulators of immunity. Nat Rev Immunol. 2008;8:478-486.

Han NR, Moon PD, Kim HM, Jeong HJ. Effect of Pyeongwee-San (KMP6) on 2,4-dinitrofluorobenzene-induced atopic dermatitis-like skin lesions in NC/Nga mice. Life Sci. 2012;90:147-153.

Han NR, Kim HM, Jeong HJ. Kanamycin activates caspase-1 in NC/Nga mice. Exp Dermatol. 2011;20:659-663.

Hansel NN, Matsui EC, Rusher R, McCormack MC, Curtin-Brosnan J, Peng RD, Mazique D, Breysse PN, Diette GB. Predicting future asthma morbidity in preschool inner-city children. J Asthma. 2011;48:797-803.

Jeong HJ, Choi Y, Kim KY, Kim MH, Kim HM. C-Kit Binding Properties of Hesperidin (a Major Component of KMP6) as a Potential Anti-Allergic Agent. PLoS One. 2011;6:e19528.

Kang TH, Han NR, Kim HM, Jeong HJ. Blockade of IL-6 secretion pathway by the sesquiterpenoid atractylenolide III. J Nat Prod. 2011;74:223-227.

Kitajima J, Kamoshita A, Ishikawa T, Takano A, Fukuda T, Isoda S, Ida Y. Glycosides of Atractylodes lancea. Chem Pharm Bull. 2003;51:673-678. 
Kimura I. Medical benefits of using natural compounds and their derivatives having multiple pharmacological actions. Yakugaku Zasshi. 2006;126:133-143.

Kobayashi K, Inohara N, Hernandez LD, Galan JE, Nunez G, Janeway CA, Medzhitov R, Flavell RA. RICK/Rip2/CARDIAK mediates signalling for receptors of the innate and adaptive immune systems. Nature. 2002;416:194-199.

Kuida K, Lippke JA, Ku G, Harding MW, Livingston DJ, Su MS, Flavell RA. Altered cytokine export and apoptosis in mice deficient in interleukin-1 beta converting enzyme. Science. 1995;267:2000-2003.

Leonard WJ. TSLP: Finally in the limelight. Nat Immunol. 2002;3:605-607.

Leung DY. Atopic dermatitis: new insights and opportunities for therapeutic intervention. J Allergy Clin Immunol. 2000;105: 860-76.

Liu YJ. Thymic stromal lymphopoietin: Master switch for allergic inflammation. J Exp Med. 2006;203:269-273.

Miller GE, Cohen S. Psychological interventions and the immune system: A meta-analytic review and critique. Health Psychology. 2001;20:47-63.

Miyata M, Hatsushika K, Ando T, Shimokawa N, Ohnuma Y, Katoh R, Suto H, Ogawa H, Masuyama K, Nakao A. Mast cell regulation of epithelial TSLP expression plays an important role in the development of allergic rhinitis. Eur J Immunol. 2008;38:1487-1492.

Miyazaki D, Nakamura T, Toda M, Cheung-Chau KW, Richardson RM, Ono SJ. Macrophage inflammatory protein-1alpha as a costimulatory signal for mast cell-mediated immediate hypersensitivity reactions. J Clin Invest. 2005;115:434-442.

Moon PD, Kim HM. Thymic stromal lymphopoietin is expressed and produced by caspase-1/NF- $\mathrm{kB}$ pathway in mast cells. Cytokine. 2011;54:239-243.

Oh HA, Kim HM, Jeong HJ. Alleviation of allergic rhinitis symptoms with Pyeongwee-San extract (KMP6). Immunopharmacol Immunotoxicol. 2012b;34:135-142.

Oh HA, Ryu JG, Cha WS, Kim HM, Jeong HJ. Therapeutic effects of traditional Korean medicine, Jeechool-Whan in allergic rhinitis model. TANG. 2012a:2:e9.
Parker-Oliver D. Asthma management: A role for social work. Health and Social work. 2005;30:167-170.

Rahman S, Sultana S. Glycyrrhizin exhibits potential chemopreventive activity on 12-O-tetradecanoyl phorbol-13-acetate-induced cutaneous oxidative stress and tumor promotion in Swiss albino mice. J Enzyme Inhib Med Chem. 2007;22:363-369.

Reed SD, Lee TA, McCrory DC. The economic burden of allergic rhinitis. Pharmacoeconomics. 2004;22:345-361.

Sakurai T, Yamada H, Saito K, Kano Y. Enzyme inhibitory activities of acetylene and sesquiterpene compounds in atractylodes rhizome. Biolo Pharm Bull. 1993;16:142-145.

Takei M, Kobayashi M, Herndon DN, Pollard RB, Suzuki F. Glycyrrhizin inhibits the manifestations of anti-inflammatory responses that appear in association with systemic inflammatory response syndrome (SIRS)-like reactions. Cytokine. 2006;35:295-301.

Tanaka K, Hasegawa J, Asamitsu K, Okamoto T. Magnolia ovovata extract and its active component magnolol prevent skin photoaging via inhibition of nuclear factor kappa B. Eur J Pharmacol. 2007;565:212-219.

Taylor BC, Zaph C, Troy AE, Du Y, Guild KJ, Comeau MR, Artis D. TSLP regulates intestinal immunity and inflammation in mouse models of helminth infection and colitis. J Exp Med. 2009;206:655-667.

Wu X, Guo R, Chen P, Wang Q, Cunningham PN. TNF induces caspase-dependent inflammation in renal endothelial cells through a Rho- and myosin light chain kinase-dependent mechanism. Am J Physiol Renal Physiol. 2009;297:F316-F326.

Yao TC, Ou LS, Yeh KW, Lee WI, Chen LC, Huang JL; PATCH Study Group. Associations of age, gender, and BMI with prevalence of allergic diseases in children: PATCH study. J Asthma. 2011;48:503-510.

Zhang K, Shan L, Rahman MS, Unruh H, Halayko AJ, Gounni AS. Constitutive and inducible thymic stromal lymphopoietin expression in human airway smooth muscle cells: role in chronic obstructive pulmonary disease. Am J Physiol Lung Cell Mol Physiol. 2007;293:L375-L382.

Ziegler SF, Artis D. Sensing the outside world: TSLP regulates barrier immunity. Nat Immunol. 2010;11:289-293. 